\title{
Helical Rim Advancement Flap with an Additional Postauricular Skin Flap: A Case Report
}

\author{
Seung Ho Kim and Jeong Hwan Choi \\ Department of Otorhinolaryngology-Head and Neck Surgery, Sanggye Paik Hospital, Inje University College of Medicine, Seoul, Korea
}

\author{
Received May 30, 2019 \\ Revised July 29, 2019 \\ Accepted August 9, 2019
}

The auricle is a three-dimensional cartilaginous frame covered with thin overlying skin. Due to its complex features, reconstructing helical rim defects after the excision of an auricular mass is challenging. Shortage of subcutaneous tissue and the presence of a tightly bound epithelium further hamper the primary closure of lateral (anterior) auricular skin defects. We present herein a case in which we used a helical rim advancement flap along with an additional postauricular skin flap. We achieved a satisfactory esthetic result with minimal loss of helical diameter and a low risk of flap necrosis by preserving the vascular network of the flap. This technique is less traumatic and will facilitate faster healing as well as improved patient recovery.

J Audiol Otol 2019;23(4):210-213

KEY WORDS: External ear · Ear auricle · Squamous cell carcinoma.

\section{Introduction}

Squamous cell carcinoma (SCCa) is a common malignant tumor that affects the head and neck area including the auricle [1]. The practice of delineating a safe resection margin during surgical excision of an auricular helical tumor inevitably results in a postoperative helical rim skin defect (HRSD) as it is difficult to obtain sufficient tissue for auricular reconstruction [2]. The shortage of subcutaneous tissue and the presence of a tightly bound epithelium further hamper the primary closure of lateral (anterior) auricular skin defects. To achieve a satisfactory esthetic result while maintaining the helical diameter, we used a 3-flap technique. In this case report, we have described the entire procedure in detail.

\section{Case Report}

An 80-year-old woman had been visiting our department for the treatment of a right auricular mass for more than 10 years. Due to the slow-growing nature of the mass, only con-

This is an Open Access article distributed under the terms of the Creative Commons Attribution Non-Commercial License (https:/creativecommons.org/licenses/by-nc/4.0/) which permits unrestricted non-commercial use, distribution, and reproduction in any medium, provided the original work is properly cited. servative care had been administered for pain relief throughout this period. A recent physical examination revealed a $1 \times 1$ $\mathrm{cm}$ sized right auricular helical mass (Fig. 1). There were no palpable neck nodes or any other abnormal findings. We performed the excisional surgery under local anesthesia and the intraoperative frozen sections revealed characteristic features of SCCa. We excised the mass with a $0.5 \mathrm{~cm}$ safety margin, including the perichondrium, causing the auricular cartilage to be exposed. No SCCa invasion was observed in the excised perichondrium.

To achieve a satisfactory esthetic result, we performed a 3-flap technique that spared the cartilage and maintained the helical length and diameter of the auricle. We created medially based bilateral advancement chondrocutaneous flaps around the defect by making parallel incisions that allowed the tissue to slide towards the defect. To avoid a dog-ear deformity, we excised the Burrow's triangle at the root of the inferior limbs of the flap. To cover the exposed cartilage, two additional triangular cutaneous flaps were elevated from the anterior surface based on the helical rim. A third advancement flap was elevated to facilitate the movement of the bilateral advancement flaps and to cover the lateral skin defect. We pulled the central chondrocutaneous flap that corresponded to the helix-scapha region and the conchal bowl area anteriorly and 
raised the medial (posterior) skin flap down to the postauricular sulcus, while keeping the posterior auricular skin intact. We undermined the posterior auricular skin (the third advancement flap) above the perichondrium down to the postauricular sulcus until the entire helix separated from the posterior skin (Fig. 2A). This flap allowed us to cover the defected posterior auricular surface without tension while maintaining the blood flow to the cartilage and the remaining tissue after closure of the wound. We trimmed the underlying helical cartilage slightly to achieve a tension-free closure. After meticulously ensuring hemostasis, we closed the wound with 5-0 nylon sutures (Fig. 2B).

The histopathological report revealed the formation of

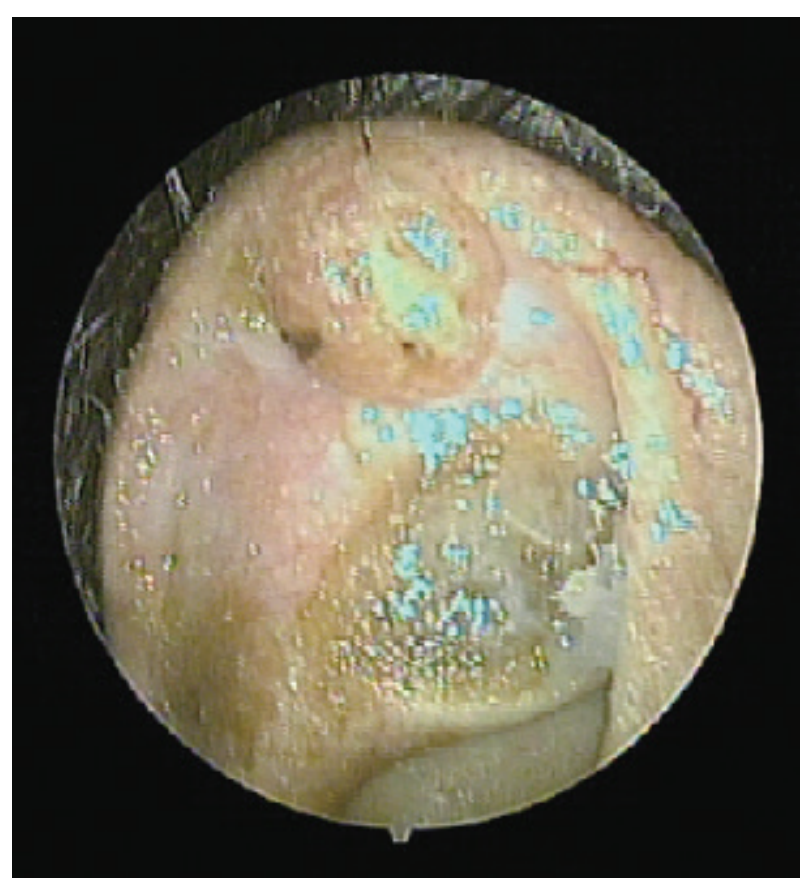

Fig. 1. Photograph of a right auricle. $1 \times 1 \mathrm{~cm}$ sized mass is observed at the right auricular helix. atypical cells with keratin pearls (Fig. 3). Postoperative complications were minor, including mild pain and ecchymosis. The esthetic outcome was satisfactory (Fig. 4).

\section{Discussion}

The auricle is a complex three-dimensional cartilaginous frame covered with thin overlying skin and little subcutaneous fat. SCCa is a common malignant tumor that affects the head and neck area, including the auricle [1]. Auricular defects have been previously reconstructed using several methods [3-5]. However, it is challenging to repair larger HRSDs properly [6]. Furthermore, as in our case, a split or full-thickness skin graft cannot be applied when the perichondrium is missing.

Closure of larger HRSDs by primary closure is rarely possible. In these cases, chondrocutaneous advancement flaps can be applied to close helical defects up to $2 \mathrm{~cm}$ in diameter $[3,4]$. Some surgical modifications were introduced to take full advantage of these flaps. Firstly, the V-Y advancement of the superior helical segment was designed [4,7] to allow increased flexibility of the flap. Then the tricone at the base of the inferior flap was excised [8] to prevent a cutaneous dog-ear deformity. Finally, cutaneous flap extensions from the anterior surface were designed to cover the exposed cartilage [9].

Apart from excising excess cartilage to avoid buckling, the third advancement flap technique can be applied to facilitate the movement of bilateral advancement flaps and to cover the lateral skin defect. The postauricular subperichondrial flap is richly vascularized by the posterior arterial network from the external carotid artery. This flap can enhance the benefits of the Antia-Buch flap by preserving natural cosmetic auricular landmarks, minimizing the loss of the helical diameter, and decreasing the risk of flap necrosis by preserving its vascular network [10].

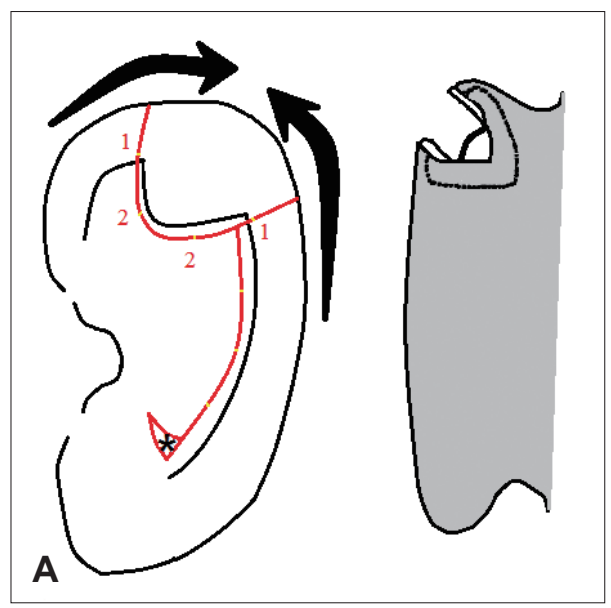

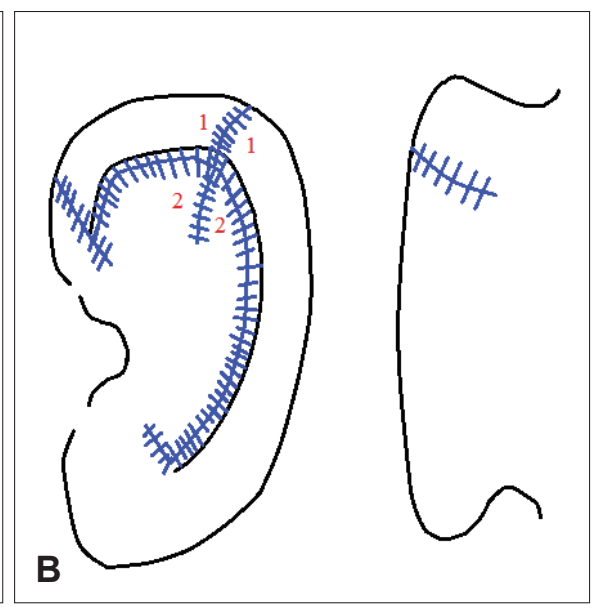

Fig. 2. Schematic diagrams of the operation. (A) Incision line for the helical rim (red line), removal of Burrow's triangle (asterisk), and movement of lobes (thick arrows). Additional third advancement flap; wide undermining of the postauricular surface (shadow area) done to cover the posterior portion of the defect. (B) Anterior and posterior view of closure (blue line). Same numbers (1 and 2) indicate counterparts of the end-toend anastomosis sides. 

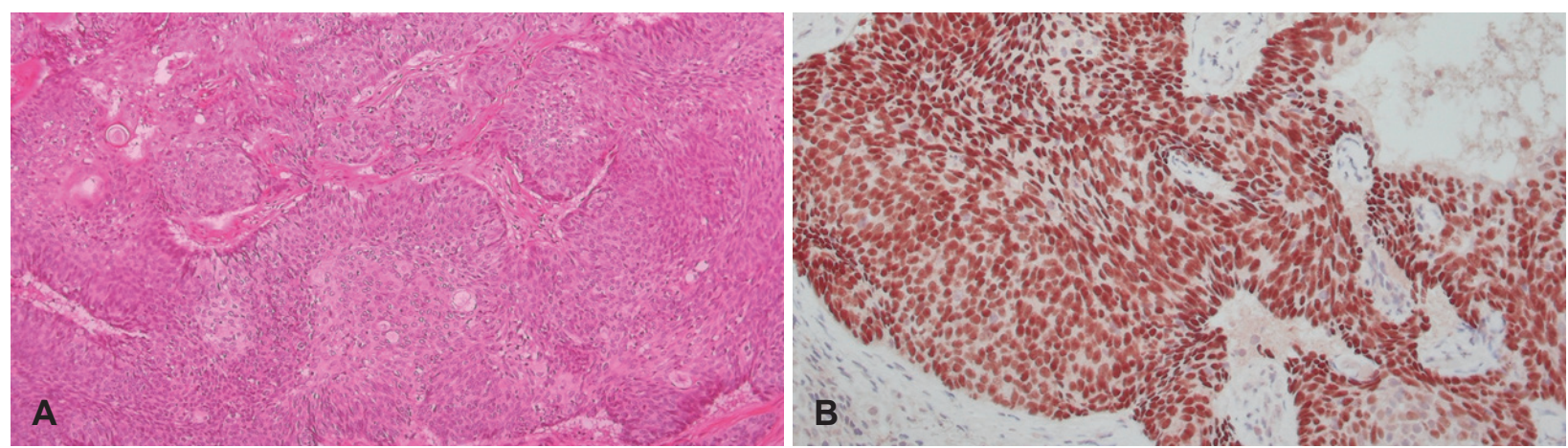

Fig. 3. Histological examination of the specimen showing keratin pearls and numerous pleomorphic nuclei (A: hematoxylin $\&$ eosin, $\times$ 100; B: immunohistochemical staining for $\mathrm{p} 63, \times 100)$.

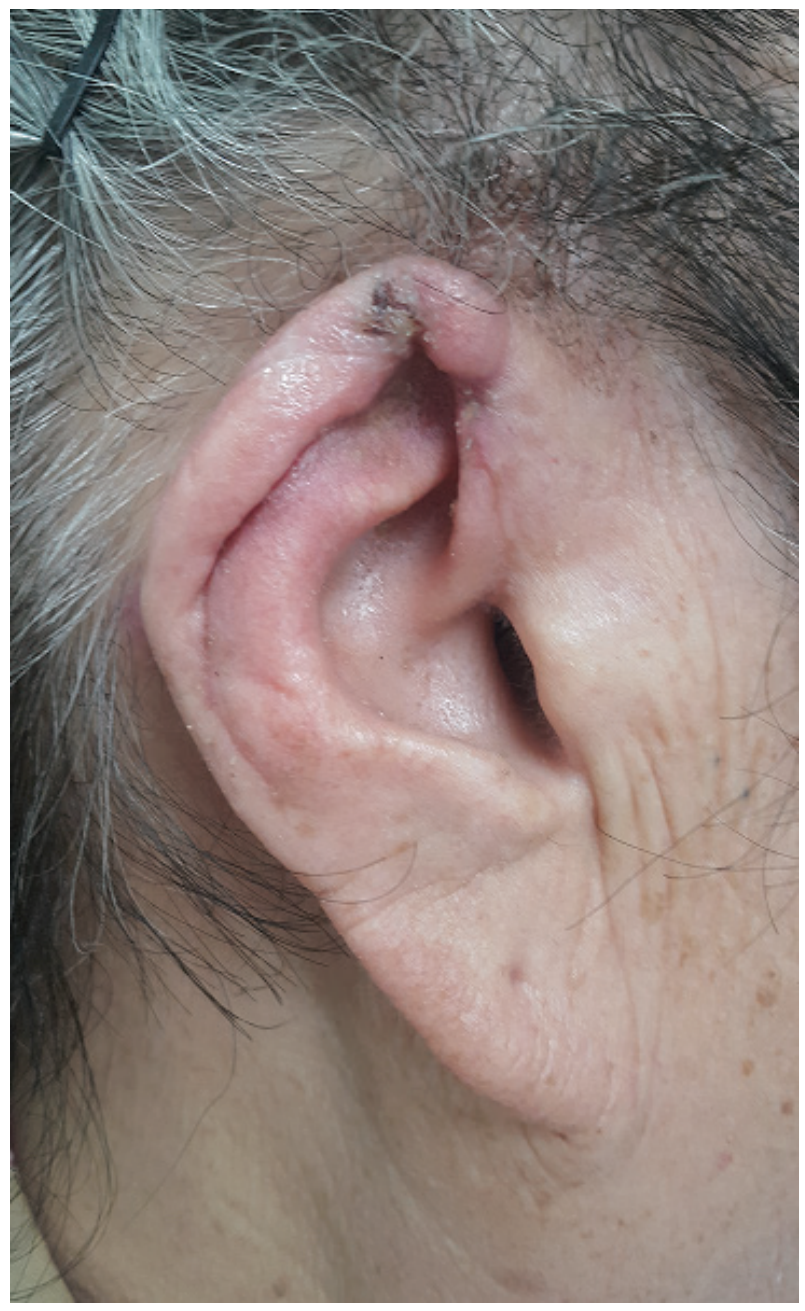

Fig. 4. Postoperative photograph of right auricle. It shows an esthetically acceptable cosmesis and minimized loss of helical diameter.

In most cases, extensive undermining from the helix to the postauricular sulcus is required to allow tension-free movement. Leaving a large dead space is inevitable; therefore, for preventing hematoma formation, meticulous hemostasis is essential. Furthermore, when the undermined flap extends to the postauricular sulcus, the greater auricular nerve should be preserved so as not to produce numbness in the auricle.

After closing the wound, we recommend that compression materials such as cottonoid or several tacking sutures be placed from the cartilage to the anterior skin surface to decrease the dead space. In spite of these advantages, there are some inevitable drawbacks to this technique. The postauricular flap often flattens the original helical convexity. Although this is not too problematic in most cases, informed consent should be obtained from the patient before the operation. Another drawback is the discrepancy in the difference between the thickness, curvature, and reflection of the upper and lower helix.

To achieve a satisfactory esthetic result, we used this 3-flap technique that spared the cartilage and minimized the loss of helical diameter. However, we preservation of the helical diameter was only possible because the mass was small with a diameter of under $2 \mathrm{~cm}$. We hope to evaluate this technique in a case involving a larger mass with a similar helical rim defect in the future.

In conclusion, the 3-flap technique is a good option in HRSD cases where the cartilage is exposed and there is no perichondrium. Bilateral advancement flaps and a third postauricular flap eliminate the need for further resection of the cartilage while preserving a normal helical rim diameter. This technique is less traumatic and facilitates healing, resulting in an improved patient recovery time.

\section{Conflicts of interest}

The authors have no financial conflicts of interest.

\section{Author Contributions}

Conceptualization: Jeong Hwan Choi. Data curation: Jeong Hwan Choi. Formal analysis: Jeong Hwan Choi. Investigation: Jeong Hwan Choi. Methodology: Jeong Hwan Choi. Project administration: Jeong Hwan Choi. Resources: Seung Ho Kim. Supervision: Jeong Hwan Choi. Validation: Jeong Hwan Choi. Visualization: Seung Ho Kim. 
Writing — original draft: All authors. Writing — review \& editing: All authors.

\section{ORCID iDs}

Seung Ho Kim

Jeong Hwan Choi

https://orcid.org/0000-0002-3588-0963

https://orcid.org/0000-0001-7348-9861

\section{REFERENCES}

1) Shockley WW, Stucker FJ Jr. Squamous cell carcinoma of the external ear: a review of 75 cases. Otolaryngol Head Neck Surg 1987;97: 308-12.

2) Choi JH, Kim JY, Yoo YS, Cho KR. Chondrocutaneous advancement flap for helical rim defect. Korean J Otorhinolaryngol-Head Neck Surg 2010;53:252-5.

3) Brent B. The acquired auricular deformity. A systematic approach to its analysis and reconstruction. Plast Reconstr Surg 1977;59:475-85.

4) Antia NH, Buch VI. Chondrocutaneous advancement flap for the marginal defect of the ear. Plast Reconstr Surg 1967;39:472-7.

5) Bumsted RM, Ceilley RI. Auricular malignant neoplasms. Identification of high-risk lesions and selection of method of reconstruction. Arch Otolaryngol 1982;108:225-31.

6) Zitelli JA. Secondary intention healing: an alternative to surgical repair. Clin Dermatol 1984:2:92-106.

7) Argamaso RV. An ideal donor site for the auricular composite graft. Br J Plast Surg 1975;28:219-21.

8) Fangman WL, Stoer CB. Reconstruction of a helical rim defect. Dermatol Surg 2010;36:245-9.

9) Low DW. Modified chondrocutaneous advancement flap for ear reconstruction. Plast Reconstr Surg 1998;102:174-7.

10) Al-Shaham AA. Helical advancement: pearls and pitfalls. Can J Plast Surg 2012;20:e28-31. 